\title{
Design and Topology Optimization of Collimation Frame for Two-Dimensional Turntable
}

\author{
Yongqiang Zhang ${ }^{1,2, a^{*}}$, Zhaohui $\mathrm{Liu}^{1, \mathrm{~b}}$ and Zhiguo $\mathrm{Li}^{1, \mathrm{c}}$ \\ ${ }^{1}$ Xi'an Institute of Optics Precision Mechanics, Xi'an 710119, China \\ ${ }^{2}$ University of Chinese Academy of Sciences, Beijing 100049, China \\ azhangyongqiang@opt.cn, 'Izh@opt.ac.cn, 'Izg@opt.ac.cn
}

Keywords: Space two-dimensional turntable, Sighting frame, Topology optimization, FEA

\begin{abstract}
Space two-dimensional turntable is the main load-bearing structure of space photoelectric detection equipment, and collimation frame is the key support component of turntable, so its stiffness characteristics are vital for the performance of turntable. Therefore, the structure of collimation frame was topologically optimized under the request of the minimum quality and stiffness maximization by using the finite element software- MSC.Patran/Nastran. Modal shape of collimation frame is regarded as the deformation of model under different incentives. The optimized results under different incentives were analyzed. Considering the aforesaid optimized results and process requirements, the lightweight design result of collimation frame was obtained. The model quality after optimization is $29.42 \mathrm{~kg}$, and this result meet the requirements of design quality. The structure after optimized is analyzed by finite element (FEA), and the result shows that the first natural frequency of collimation frame is $169 \mathrm{~Hz}$, which satisfies the requirement of structure design. Finally, the modal experiment of structure was conducted, and the results verify that the result of finite element analysis is correct.
\end{abstract}

\section{Introduction}

Space two-dimensional turntable is the payloads of satellite. It provides installation platform and satisfies the camera's two-dimensional point accuracy. It will experience severe mechanical environments such as vibration impact and space microgravity from launch to working on the orbit. These factors require that the turntable has enough rigidity. Turntable shafting is divided into pitch axis and azimuth axis. The collimation frame, not only the vital support component for pitch axis and detection equipment, but also the rotary structure for azimuth axis, determines the stiffness properties of the turntable at great degree. Therefore, optimizing the design must to be done on the collimation frame. In this way it can get appropriate stiffness and dynamic performance to avoid structure resonance on the premise of quality as light as possible. The size of the collimation frame studied in the paper is $800 \mathrm{~mm} \times 250 \mathrm{~mm} \times 700 \mathrm{~mm}$, and the quality is no more than $30 \mathrm{~kg}$, and the fundamental frequency is greater than $150 \mathrm{~Hz}$. Topology optimization is an important method for structure optimization design. It has been widely applied to the lightweight design of structure. The space camera framework is designed by using the method of topology optimization by Li et al. [1]. Infrared seeker stabilized platform main frame is designed by using the method of topology optimization by Liu et al. [2].

With the rapid development of computer technology in recent years, the finite element analysis has been widely used in engineering. A large number of excellent finite element analysis software such as ANSYS and MSC.Patran/Nastran has become an effective way to solve the problem of engineering analysis and calculation. MSC.Patran, earliest developed by NASA, is a powerful parallel frame type finite element analysis system. MSC.Nastran is solver. They were widely used in aerospace industry [3]. The design of collimation frame should accord with the requirement of practical process. In the paper, the collimation frame is designed by using the method of topology optimization. The variable density method and the finite element analysis software MSC.Patran /nastran were been used to design the collimation frame. 


\section{Topology Optimization Theory}

The main methods of structure optimization can be divided into three types such as size optimization, shape optimization and topology optimization [4]. Topology optimization is a optimal design method which sets the corresponding constraints according to the load distribution, optimizes the distribution of structural material as to achieve the optimum of the objective function [5].

In fact, topology Optimization is the theory of space distribution optimization for material in the design space. Variable density method is the most common method for topology optimization. The variable density method was put forward by Mlejnek [6]. Its core idea is that a pseudo density material is assumed whose density distribution is from zero to one. Discrete variable optimization problem is turned into continuous distribution optimization problem between 0 and 1 by using density interpolation function. The parts whose density values are lower than floor level will be deleted gradually [7]. In order to make the middle density material rapidly converge to the border, penalty factor is introduced. Penalty factor is vital in that material interpolation model determines the function relationship between the elastic modulus and pseudo density for structural unit. The material interpolation models include two types.

SIMP model

$$
\mathrm{E}=\rho^{p} E_{0}
$$

RAMP model

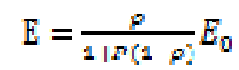

Mathematical model of structural topology optimization includes three essential factor such as design variable, objective function and constraint conditions. In the variable density method, density distribution is the design variable, the lightest structure quality or the biggest structure stiffness is objective function normally, and Volume percentage of the optimization is the constraint condition.

Mathematical model of stiffness optimization is that

$$
\begin{aligned}
& \text { find: } \rho=\left(\rho_{1}, \rho_{2}, \ldots, \rho_{n}\right)^{T} \\
& \text { min: } C-F^{T} U-U^{\top} K U \\
& \text { subject: } \frac{\| \varphi)}{V_{i}} \leq f \\
& K U=F \\
& 0<\rho_{\min } \leq \rho_{i} \leq 1
\end{aligned}
$$

where, $\mathrm{C}$ is value of smooth degree, and $\mathrm{K}$ is total stiffness matrix of the structure, and $\mathrm{U}$ is total displacement vector, and V is volume of structure. Optimization system of structure is constituted by topology optimization, size optimization and shape optimization. They each are very important in different stages.

\section{Design and Topology Optimization of Collimation Frame}

The collimation frame is not only the vital support component for pitch axis and detection equipment, but also the rotary structure for azimuth axis. The initial shape of the collimation frame is shown in Fig. 1. Its dimension is $800 \mathrm{~mm} \times 250 \mathrm{~mm} \times 700 \mathrm{~mm}$. 


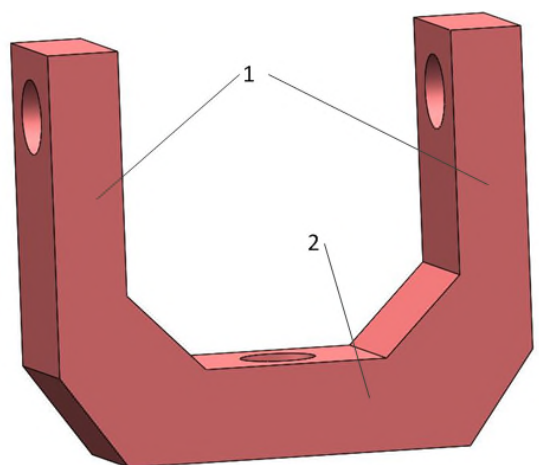

1. Two arms of collimation frame 2. Pedestal of collimation frame

Fig. 1 Rough shape of collimation frame.

Finite Element Modeling. The finite element model of the collimation frame is established by Patran, and then calculated by Nastran. Because the collimation frame is symmetrical structure, only half of it is established by hexahedral mesh, so as to improve the computational efficiency. Finite element model is shown in Fig. 2. Titanium alloy (ZTC4) is chosen as the material of the collimation frame. Its elastic modulus is $118 \mathrm{GPa}$, and Poisson's ratio is 0.33 , and the density is 4550 $\mathrm{kg} / \mathrm{m}^{3}$.

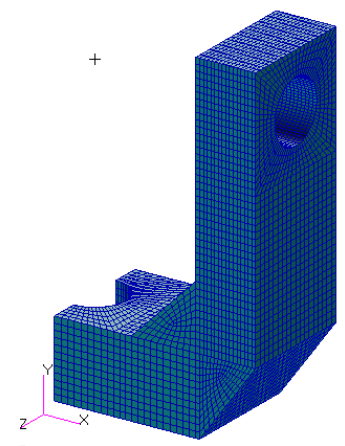

Fig. 2 Mesh model of collimation frame.

Topology Optimization Process. Classic topology optimization process mainly contains the determination of the optimization area, constraint condition, and objective function. The optimization area is all entity units except the units near the axle holes, for the reason that the hole of azimuth axis is fixed and the hole of pitch axis is imposed by force. The constraint condition is residual volume ratio, forty percent is often selected. The objective function is the maximum stiffness. In other words, we select the minimum value of the smooth degree as the optimization goal in the Patran. The supporting position of the collimation frame is the hole of azimuth axis, so fixed constraint is create on it. Swing and reverse of the collimation frame arms are main modal vibration modes, now it is equivalent to structural deformation under the different forces and torques. In the paper, the forces along the $\mathrm{X}, \mathrm{Y}$ and $\mathrm{Z}$ axis and the torque Around the $\mathrm{Y}$ axis are put on the hole of pitch axis respectively.

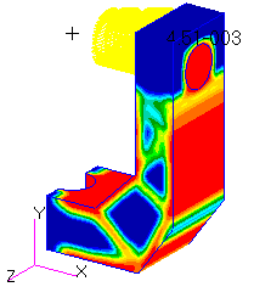

a. X direction

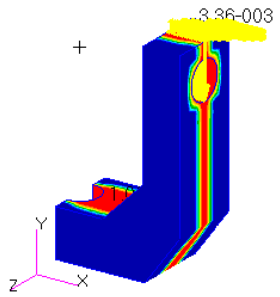

b. Y direction

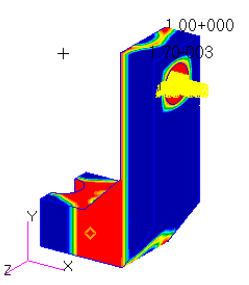

c. $\mathrm{Z}$ direction

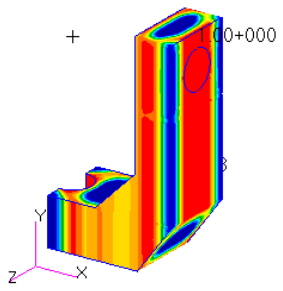

d. the torque

Fig. 3 Optimal results of model under different forces and torque. 
Optimization Results. The optimization results of the model under different forces and torque is shown in the Fig. 3. As shown in the Fig. 3, the red part is the main material distribution area where should be kept, because it is the main support area in the model. The blue part is useless area where almost don't support the static load and should be deleted. Considering the above several kinds of optimization results and the feasibility of processing technology, the geometry optimization model of the collimation frame is created by using Solidworks. The model is shown in Fig. 4. The quality of the optimization model is $29.42 \mathrm{~kg}$, which satisfies design requirement.

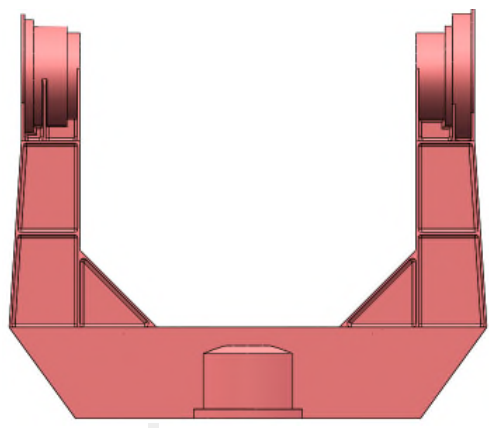

a. Main view

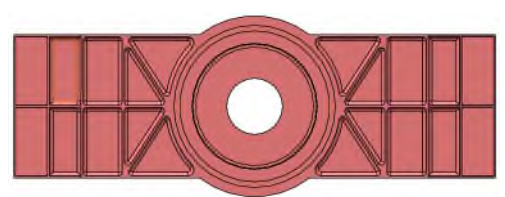

b. Bottom view

Fig. 4 Optimal model of collimation frame

\section{Modal Analysis of Collimation Frame}

Finite Element Modal Analysis. Finite element model of collimation frame is built by software MSC.Patran and analyzed by MSC.Nastran. The model is meshed by Tet10 mesh [8]. The finite element model is shown in Fig. 5. Titanium alloy (TC4) selected before is used as material of the model, and the material poisson ratio is 0.33 .

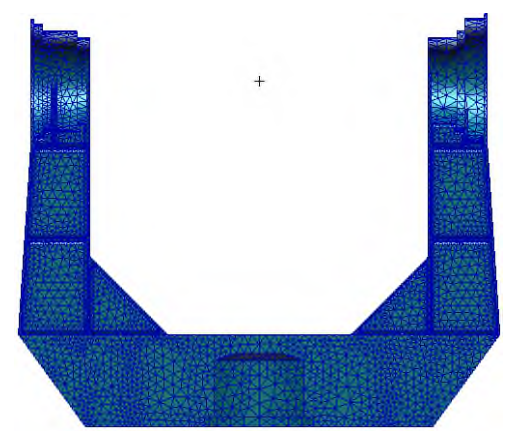

Fig. 5 FE model of collimation frame.

The first 5-order nonzero modal frequencies are shown in Table 1. The first modal vibration shapes are shown in Fig. 6.

Table 1. Results of finite element modal analysis for collimation frame.

\begin{tabular}{ccc}
\hline Mode No. & Frequency/Hz & Mode shape \\
\hline 1 & 169.5 & Bend \\
2 & 220.7 & Bend \\
3 & 405.4 & Torsion \\
4 & 432.0 & Bend \\
5 & 451.3 & Torsion \\
\hline
\end{tabular}




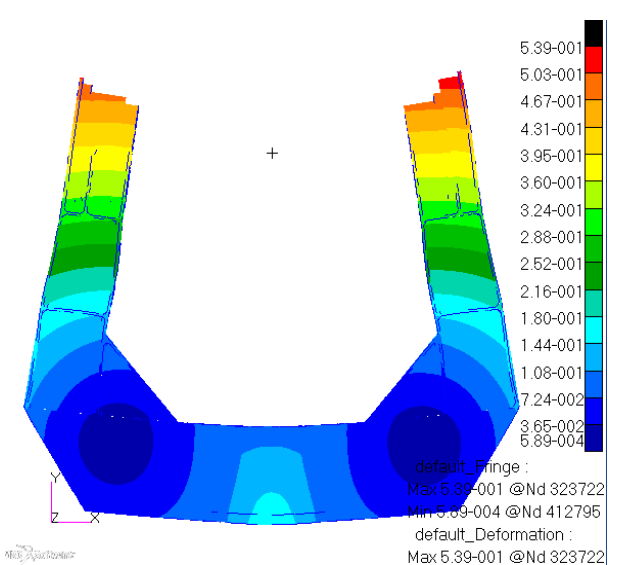

Fig. 6 The first modal vibration shapes.

Table 1 show that the first nonzero frequency of model is $169.5 \mathrm{~Hz}$, which is greater than $150 \mathrm{~Hz}$ and satisfies the design requirement. As shown in the Fig.6, it is evident that deformation areas of model are mainly concentrated in two arms, so the stiffness of the two arms, especially the roots, should be strengthened.

Experiment Modal Analysis. It is common to combine experimental modal analysis with finite element analysis. Experimental modal analysis is a method that is based on the input and output of the system. It identifies the modal parameters by estimating the frequency response function and impulse response function, namely obtain structural modal parameters such as system poles, inherent frequencies, modal damping factor, etc. [9].

32 channel data acquisition system and Smart Office Analyzer data processing analysis software is used as modal test equipment to acquire, process and analyze the data. The structure is excited by hammer and responded by acceleration sensor. Modal parameter identification is realized by collecting the frequency response function data curves of each point. The connectivity of the transducer is shown in Fig.7.

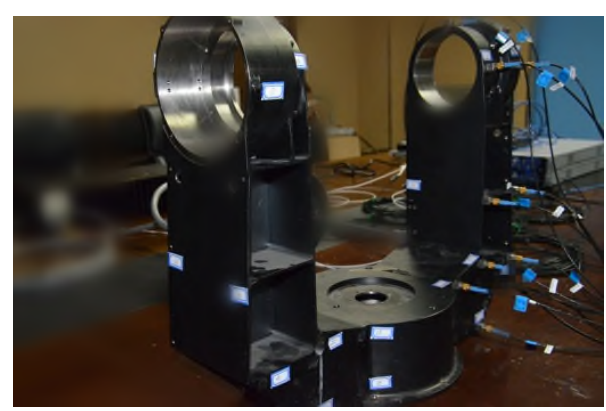

Fig. 7 Layout of the transducer.

The first 5-order inherent frequency of the modal test results compared with the finite element simulation results as shown in Table 2.

Table 2. Modal test and finite element simulation results.

\begin{tabular}{ccccc}
\hline Order & Mode shape & Test frequency[Hz] & FEA frequency[Hz] & Deviation \\
\hline 1 & Bend & 171.8973 & 169.5 & $1.4 \%$ \\
2 & Bend & 219.8616 & 220.7 & 0 \\
3 & Torsion & 395.4537 & 405.4 & $2.5 \%$ \\
4 & Bend & 421.9651 & 432.0 & $2.4 \%$ \\
5 & Torsion & 435.0493 & 451.3 & $3.7 \%$ \\
\hline
\end{tabular}


The figures in Table 2 indicate that the results of experimental modal analysis and finite element analysis are almost identical. The deviation of the first 5-order natural frequencies is less than 5 percent and corresponding vibration mode completely consistent with the result of analysis, which shows that the results of finite element analysis are correct.

\section{Summary}

Topology optimization design of the collimation frame is realized by using MSC. Patran /Nastran. The quality of the optimization model is $29.42 \mathrm{~kg}$, which satisfies design requirement.

3D model of collimation frame is built by Solidworks. Its modal analysis is realized by using MSC. Patran /Nastran. It turned out that the first order natural frequency is $169.5 \mathrm{~Hz}$, which is greater than $150 \mathrm{~Hz}$ and satisfies the stiffness design requirement.

Well-designed collimation frame is processed with requested material. Then experimental modal analysis of structure was carried out. The results show that deviation of the first 5-order natural frequencies are less than 5 percent, and corresponding vibration modes are completely consistent, which proves that the results of finite element analysis are reasonable. Therefore, the design of collimation frame is feasible. It has profound guiding significance for engineering practical problem.

\section{References}

[1] C. Li, X. He, Q. Liu. Design and topology optimization of space camera frame fabricated by high volume fraction SiC/Al composite material, Infr. Laser Eng. 43(8) (2014) 2526-2531.

[2] Z. Y. Liu, T. Zhang, P. Wang, et al. Topology optimization design for main frame of infrared seeker's stabilization platform, Infr. Laser Eng. 45(2) (2016) 218001-0218001(5).

[3] J. J. Yuan, S. Y. Chen, H. Huang, Engineering applications of structural optimization system based on Patran/Nastran, J. Beijing University of Aeronaut. Astronaut. 02 (2006) 125-129.

[4] T. X. Xia, W. X. Yao, A survey of topology optimization of continuum structure, Adv. Aeronaut. Sci. Eng. 01 (2011) 1-11+55.

[5] H. A. Eschenauer, N. Olhoff, Topology optimization of continuum structures: A review, Appl. Mech. Rev. 54(4) (2001) 1453-1457.

[6] H. P. Mlejnek, R. Schirrmacher, An engineer's approach to optimal material distribution and shape finding, Comput. Meth. Appl. Mech. Eng. 106(1-2) (1993) 1-26.

[7] L. Liu, M. H. Gao, L. F. Li, et al. Primary mirror topological optimum design of space camera, Infr. Laser Eng. 06 (2010) 1066-1069.

[8] K. Long, C. Z. Jia, B. F. Li, et al. Patran 2010 and Nastran 2010 finite element analysis from entry to the master, China Machine Press, China, 2011.

[9] J. Y. Yang, X. Y. Wang, Experiment Mode Analysis, Beijing Institute Technology Press, Beijing, 1990. 